\title{
The study on copy number alteration of clear cell renal cancer in Chinese population
}

\author{
Ning Zhang ${ }^{*}$, Siteng Chen ${ }^{2 *}$, Guangliang Jiang ${ }^{1}$, Yishuo $\mathrm{Wu}^{3}$, Jialiang Shao ${ }^{2}$, Wennuan Liu ${ }^{4}$, Xiang Wang ${ }^{2 \bowtie}$, \\ Rong $\mathrm{Na}^{1}$, and Jianfeng $\mathrm{Xu}^{3,4}$ \\ 1. Department of Urology, Ruijin Hospital, Shanghai Jiao Tong University School of Medicine, Shanghai, China \\ 2. Department of Urology, Shanghai Gerneral Hospital, Shanghai Jiao Tong University School of Medicine, Shanghai, China \\ 3. Department of Urology, Huashan Hospital, Fudan University, Shanghai, China \\ 4. Program for Personalized Cancer Care, Northshore University HealthSystem, Chicago, IL 60201, USA \\ *Equal contributors and co-first authors \\ $\square$ Corresponding authors: Shanghai Jiao Tong University School of Medicine, Shanghai, China; Rong Na, Department of Urology, Ruijin Hospital, Email: \\ narong.hs@gmail.com (R.N.); Xiang Wang, Department of Urology, Shanghai Gerneral Hospital, Email: seanw_hs@163.com (X.W.)
}

(c) The author(s). This is an open access article distributed under the terms of the Creative Commons Attribution License (https://creativecommons.org/licenses/by/4.0/). See http://ivyspring.com/terms for full terms and conditions.

Received: 2019.01.19; Accepted: 2019.08.16; Published: 2020.01.01

\begin{abstract}
Objectives: Copy number alteration (CNA) is one of the important genetic variations. Although there are many studies on renal cancer CNA, few studies are based on the Chinese population. In our study, our objective is to acquire the whole-genome CNA landscape in Chinese population and explore the tumor risk-associated functional genes in the CNA regions, by detecting whole-genome in the clear cell renal cancer ( $c c R C C)$ tissues.

Methods: We enrolled 35 formalin fixed paraffin embedded samples, which were processed by Oncoscan assay, and then acquired the data of whole-genome CNA. Then genes annotation and enrichment analyzing were processed. Furthermore, the gene burden and the affected bp (base pair) per Mbp (million bp) regions in whole-genome were analyzed by comparison of different $\mathrm{T}$ stage affected by CNA.

Results: We acquired the whole-genome CNA landscape by Oncoscan detection, and found out the high-frequency CNA regions which were not reported in previous studies, for example, 11P11, $22 q 11.23,20 q 11.3$ (PDRG1), and Xp22.33 so on. During the analyzing of genes annotation and enrichment, we found out some ccRCC functional genes in the CNA regions which might play a role in the biological process, for example, the copy number loss of DNA repair genes (TTC5、PARP2, etc.) and tumor suppressor genes (TADA3, VHL, BAP1, ERC2-IT1, etc.), the copy number gain of oncogenes (ABL2, MET, HUWE1, etc.) and Notch signal pathway genes (MDK, etc.). Besides, gene fusion (GSTTP and GSTTP2) was noticed at 22q11.23 which copy number loss occurred, and the frequency is $46 \%$. And between the different T stage patients affected by CNA, the T2+T3 group carried more high-frequency CNA regions ( $P$-value was 0.012 ).

Conclusions: In this study, the whole-genome ccRCC CNA landscape in Chinese population was acquired, a few functional genes and fusion genes were found out. However, a larger scale of samples is still needed to validate our results.
\end{abstract}

Key words: ccRCC, copy number alteration, Oncoscan, enrichment, gene burden, Chinese

\section{Introduction}

Renal cell cancer (RCC) represents $2 \% \sim 3 \%$ of all cancers, and about $75 \%$ of RCC are clear cell renal cancer (ccRCC) [1]. The estimated new cases and deaths were 63,990 and 14,400 in the US in 2017 , and the number of new cases and deaths of renal cancer respectively were 15.6 per 100,000 and 3.9 per 100,000 
men and women per year [2]. In China, It was predicted that there would be about 66,800 newly diagnosed renal cancer cases and 23,400 deaths in 2015, namely 5.3 per 100,000 and 1.8 per 100,000 new cases and deaths [3]. Despite the incidence rates of RCC stabilized during these years, due to the relatively high incidence, RCC has become one of the most important healthcare issues worldwide.

Copy number alteration (CNA) is one of the important genetic variations, whose regions of variation range from $1 \mathrm{kbp}$ to $1 \mathrm{mbp}$, and is widely distributed in the human genome. Besides, some functional genes included in CNA maybe play a key role in the tumorigenesis, prognosis and response to drug treatment [4]. For example, the copy number loss (CN loss) of tumor suppressor genes would affect the regulation of cell proliferation, leading to uncontrollable proliferation, and then cause cancer. In addition, the copy number gain ( $\mathrm{CN}$ gain) of oncogenes also results in tumor occurrence, because of the accumulation of oncogenesis effect [5]. Although there are many studies on RCC CNA, few studies are based on the Chinese population. In this study, our objective is to acquire the whole genome CNA landscape in Chinese population and explore the tumor risk-associated functional genes in the CNA regions, by detecting the whole genome in the ccRCC tissues.

\section{Materials and Methods}

\section{Patients and tissues}

All ccRCC specimens were from Huashan hospital, Fudan University. The inclusion criteria were: (1) Patients received radical nephrectomy or partial nephrectomy via open procedure or laparoscopic procedure because of kidney tumor; (2) the specimens were diagnosed with ccRCC by department of Pathology in our hospital (all the specimens were reviewed by the same group of pathologists.); (3) Clinical information was collected. Patients who were diagnosed with other types of kidney tumor, had missing clinical information were excluded. Finally, 35 FFPE (Formalin Fixed Paraffin Embedded) samples were included. The study was approved by the institutional review board of Huashan Hospital, Fudan University, Shanghai, China.

\section{The whole genome in the cCRCC and Statistical Analysis}

The whole-genome DNA was isolated and purified from FFPE samples using QIAamp DNA FFPE Tissue Kit (QIAGEN), and was quantified using the Quant-iT ${ }^{\mathrm{TM}}$ PicoGreen ${ }^{\circledR}$ dsDNA Assay Kit (Life
Technologies). Then, the DNA was processed by use of Oncoscan (Affymetrix Inc) assay, and array fluorescence intensity data (CEL files), generated by Affymetrix ${ }^{\circledR} \quad$ GeneChip ${ }^{\circledR} \quad$ Command Console ${ }^{\circledR}$ (AGCC) Software version 4.0 were processed using OncoScan ${ }^{\circledR}$ Console software to produce OSCHP files and a set of QC metrics (inclusion criteria: MAPD $\leq 0.3$ and ndSNPQC $\geq 26$ ). And then, the OSCHP files were analyzed and displayed by the software Nexus Express for Oncoscan 3.0 [6]. Finally, the visible data and whole genome CNA landscape were acquired, and the CNA frequency was calculated by Nexus Express for Oncoscan 3.0. Besides, genes annotation and enrichment analyzing were processed by the method of BP (Biological Process), GO (Gene ontology) in the DAVID database (the Database for Annotation, Visualization and Integrated Discovery), and the significant threshold was set as $P$-value $<0.05$. Then the functional genes related to biology process of oncogenesis in the CNA regions were further explored. Furthermore, the heatmap was conducted by R program (version 3.2.2). The SPSS (version 22) was used to analyze the gene burden and the affected base pair (bp) per million bp (Mbp) regions in the whole genome by comparison of different $\mathrm{T}$ stage affected by CNA.

\section{Results}

\section{Baseline characteristics of the patients}

In the Oncoscan assay, a total of 35 ccRCC samples were finally included in this study, of which $21(60 \%)$ were male and $14(40 \%)$ were female. The mean age of the cases was $57.6 \pm 9.6$ and the mean body mass index (BMI) was 24.46 \pm 3.1 . According to the TNM stage, there were 22 patients $(63 \%)$ with T1a stage (tumor size $\leq 4 \mathrm{~cm}$ ), 6 patients $(17 \%)$ with $\mathrm{T} 1 \mathrm{~b}$ stage $(4 \mathrm{~cm}<$ tumor size $\leq 7 \mathrm{~cm}), 4$ patients $(11 \%)$ with T2a stage $(7 \mathrm{~cm}<$ tumor size $\leq 10 \mathrm{~cm}), 2$ patients $(6 \%)$ with $\mathrm{T} 2 \mathrm{~b}$ stage (tumor size $>10 \mathrm{~cm}$, but tumor confined to kidney), 1 patients (3\%) with T3a stage (tumor grossly extends into the renal vein) (Table 1$)$.

\section{Landscape of whole-genome CNA in Chinese population}

The whole-genome CNA were detected in 35 ccRCC FFPE samples using Oncoscan assay. The software Nexus Express for Oncoscan 3.0 was used to analyze the raw data, generate the visible data, and depict the landscape of whole-genome CNA (Fig. 1A and Fig. 1B). In this CNA landscape, the red and blue areas respectively represented $\mathrm{CN}$ loss and $\mathrm{CN}$ gain, and the statue of whole-genome CNA in 24 chromosomes of 35 ccRCC samples was observed clearly. For example, we could notice the obvious $\mathrm{CN}$ 
loss in the whole chromosome $3 p$, partial chromosome $8 p$ and chromosome $22 q$, and the frequencies of occurrence were high. Besides, we could notice the $\mathrm{CN}$ gain regions of high frequencies in chromosome $5 q$, chromosome Xq and Xp. Furthermore, the heatmap (Oncoprint) was depicted based on CNA regions of high frequency and representative functional genes (Fig. 2). This heatmap showed $\mathrm{CN}$ losses (blue) and gains (red) in 35 samples with Ocoscan assay data, and the affected genes in each locus that harbor CNA were listed in the parentheses. In this heatmap, the left histograms showed percentages of affected samples.

Based on the outcomes of Ocoscan assay in our study, we chose some CNA regions which had high frequency $(\geq 20 \%)$ to make comparison with the Cancer Genome Atlas (TCGA) database on CNA associated with ccRCC (Table 2). In the first four columns of Table 2, we listed the high-frequency CNA regions we found from Ocoscan assay, among which the $\mathrm{CN}$ gain in 11p11 (MDK), 22q11.23 (PLA2G6), Xp22.33 (XGY3), and the CN loss in 20q11.3 (PDRG1), 16p11.2-p11.1 (SMG1P2), were found for the first time. Compared with TCGA database, the CNA frequency of some regions in Chinese was obviously higher, for example, 3p25.3 (VHL), 5q35.3 (SQSTM1), 13q21 (RB1), 17q21.33 (ABCC3), and Xq28 (SPRY3) so on. So the functional genes located in these $\mathrm{CNA}$ regions had a potential to become the RCC biomarkers. In the
Supplementary Table 1, more CNA regions in Chinse were compared with TCGA cohort.

Table 1. Demographic and clinical information of study population

\begin{tabular}{ll}
\hline Variables & ccRCC $(\mathrm{n}=35)$ \\
\hline No. of cases & 35 \\
Age (yr), Median (range) & $55(40-85)$ \\
Gender (n, \%) & \\
$\quad$ Male & $21(60 \%)$ \\
Female & $14(40 \%)$ \\
Tumor size (cm), Median (Range) & $3.5(2-13)$ \\
T Stage (n, \%) & \\
T1a & $22(63 \%)$ \\
T1b & $6(17 \%)$ \\
T2a & $4(11 \%)$ \\
T2b & $2(6 \%)$ \\
T3a & $1(3 \%)$ \\
N stage (n, \%) & \\
N0 & $35(100 \%)$ \\
N1 & 0 \\
M stage (n, \%) & \\
M0 & $35(100 \%)$ \\
M1 & 0 \\
Furhman (n, \%) & \\
I & $1(3 \%)$ \\
II & $17(48 \%)$ \\
III & $3(9 \%)$ \\
IV & 0 \\
Missing & $14(40 \%)$ \\
\hline
\end{tabular}

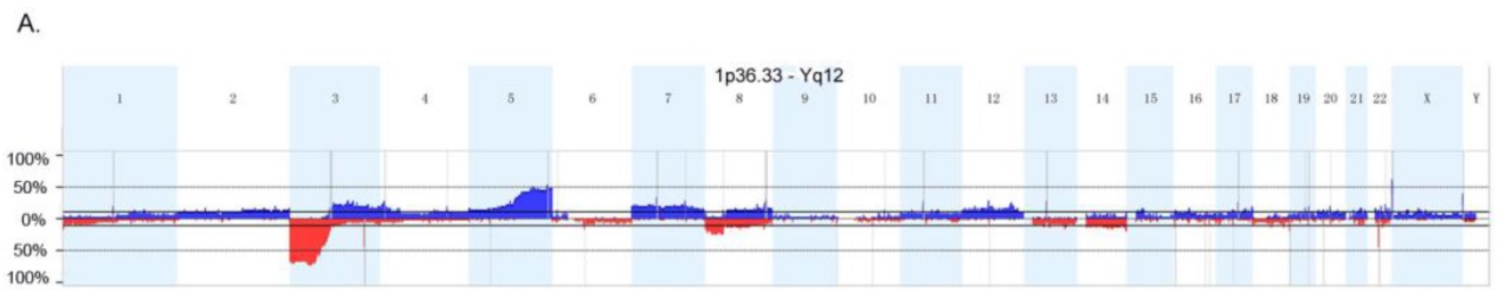

B.

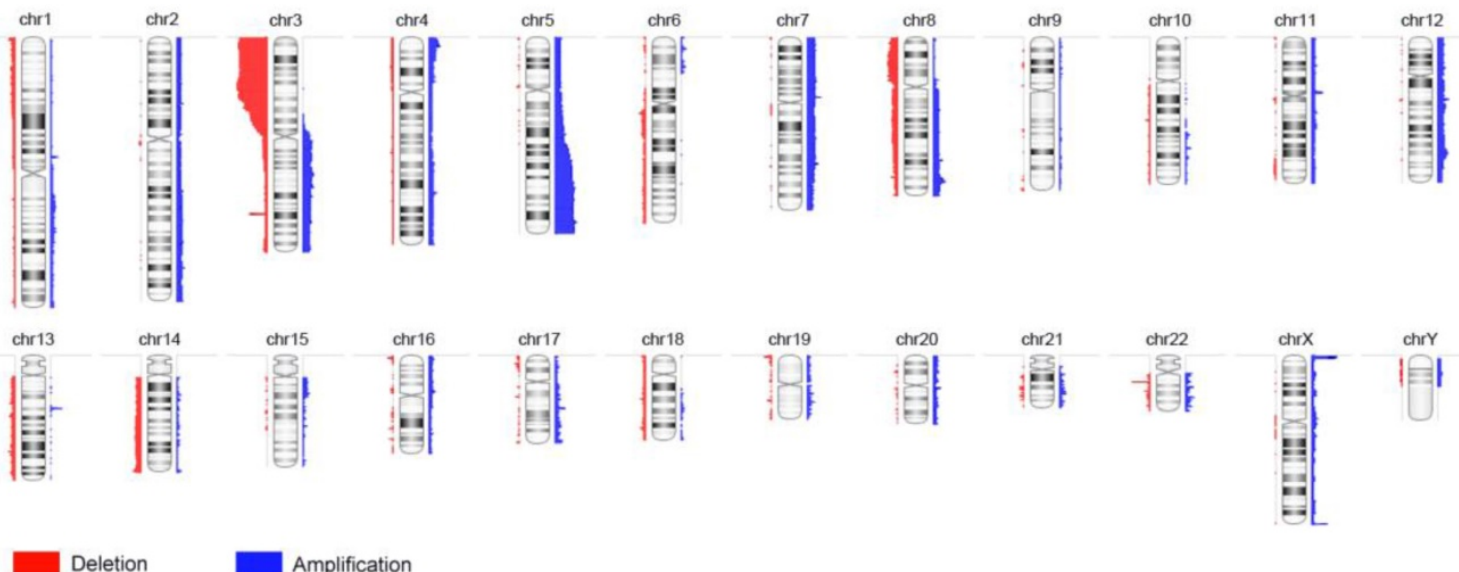

Figure 1. Landscape of whole-genome copy number alteration (CNA). The red and blue areas respectively represented copy number loss and copy number gain, and the statue of whole-genome CNA in 24 chromosomes of 35 clear cell renal cancer samples was observed clearly. 


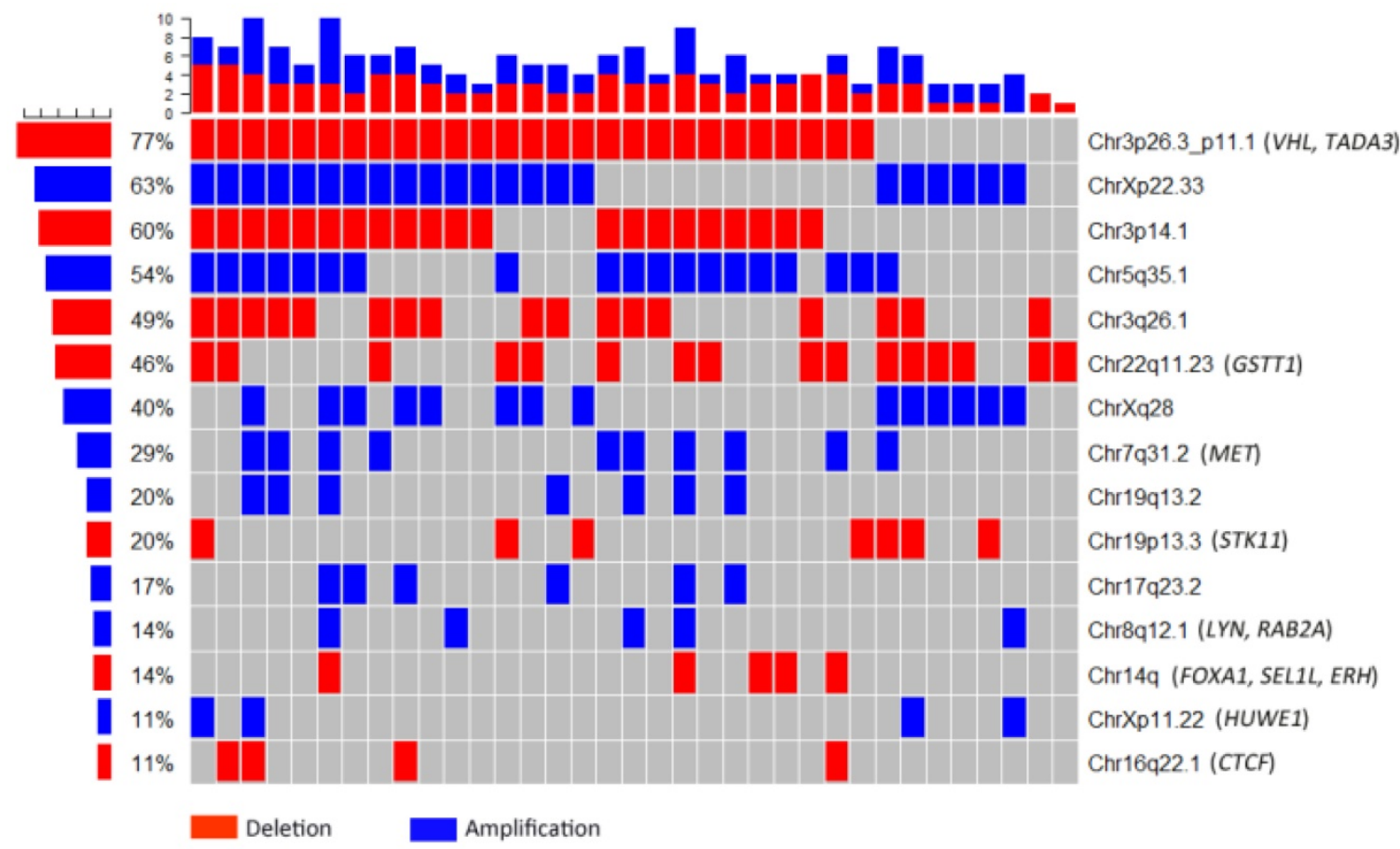

Figure 2. Heatmap based on copy number alteration regions of high frequency and representative functional genes. The red and blue areas respectively represented copy number loss and copy number gain. The left histograms showed percentages of affected samples.

Table 2. CNAs of $c c R C C$ with highest frequencies $(\geq 20 \%)$ in Chinese and the comparison with TCGA cohort (mainly European Americans)

\begin{tabular}{|c|c|c|c|c|c|c|}
\hline \multirow[t]{2}{*}{ Chr } & \multirow[t]{2}{*}{ Region } & \multicolumn{2}{|c|}{ Chinese $(n=35)$} & \multicolumn{2}{|c|}{ TCGA $(n=537)$} & \multirow[t]{2}{*}{ Affected Genes } \\
\hline & & Event & Frequency & Event & Frequency & \\
\hline \multirow[t]{5}{*}{3} & p26.2 & Del & $48.57 \%$ & Del & $91 \%$ & MECOM \\
\hline & p25.3 & Del & $71.43 \%$ & Del & $11 \%$ & VHL \\
\hline & p22-p21 & Del & $74.29 \%$ & Del & $89-92 \%$ & BAP1, SETD2 \\
\hline & p12.1 & Del & $31.43 \%$ & Del & $51 \%$ & CADM2 \\
\hline & q21 & Amp & $31.43 \%$ & Amp & $0.4 \%$ & CNBP \\
\hline \multirow[t]{2}{*}{5} & $\mathrm{q} 31.2$ & Amp & $51.43 \%$ & Amp & $14 \%$ & EGR1, PKD2L2 \\
\hline & q35.3 & Amp & $48.57 \%$ & Amp & $15 \%$ & SQSTM1 \\
\hline 7 & $\mathrm{q} 22.1$ & Amp & $20.00 \%$ & Amp & $0.7 \%$ & RADIL, TRRAP, ZAN \\
\hline 8 & $\mathrm{q} 24.21$ & Amp & $25.71 \%$ & Amp & $15 \%$ & MYC \\
\hline 11 & p11 & Amp & $31.43 \%$ & - & - & OR4C12,MDK \\
\hline 13 & q21 & Del & $22.86 \%$ & Del & $0.20 \%$ & RB1 \\
\hline 14 & $\mathrm{q}$ & Del & $51.43 \%$ & Del & $45 \%$ & HIF1A,NRXN3 \\
\hline 16 & p11.2-p11.1 & $\mathrm{LOH}$ & $100 \%$ & - & - & SMG1P2 \\
\hline 17 & q21.33 & Amp & $31.43 \%$ & Amp & $0.40 \%$ & $\mathrm{ABCC} 3$ \\
\hline 19 & q13.2 & Amp & $28.57 \%$ & Amp & $0.20 \%$ & WTIP \\
\hline 20 & q11.3 & Del & $20.00 \%$ & - & - & PDRG1 \\
\hline 22 & q11.23 & Amp & $45.71 \%$ & - & - & PLA2G6 \\
\hline \multirow[t]{2}{*}{$X$} & p22.33 & Amp & $62.86 \%$ & - & - & XGY2 \\
\hline & q28 & Amp & $40 \%$ & Amp & $0.80 \%$ & SPRY3 \\
\hline
\end{tabular}

CNA, copy number alteration; ccRCC, clear cell renal cancer; TCGA, the Cancer Genome Atlas; Chr, chromosome; Del, delete; LOH, loss of heterozygosity; Amp, amplification.

\section{Genes functional annotation and enrichment}

For the further study on the CNA regions and the genes located in these regions, 4437 genes which were located in the CNA regions (the frequency $\geq 10 \%$ ) were included to conduct the analyzing of genes annotation and enrichment by the method of biological process in the Database for Annotation, Visualization and Integrated Discovery (DAVID).
During this process, we set the significant threshold as $P$-value $<0.05$, and acquired a few potential biological processes and functional genes related to the oncogenesis and tumor progression (Fig. 3). For example, the functional genes which were enriched in the Wnt signal pathway (PSME2, PSMB11, PSMB5) and Notch signal pathway (FOXA1, MMP14, MDK), DNA repair pathway (REC8, DDB2, FAN1, TTC5, etc.), DNA modification pathway (DNMT3A, ASCC1, 
CTCF, etc.). Furthermore, these functional genes in different pathways which were significantly enriched were compared with TCGA database (Table 3). Finally, some higher-frequency functional genes and new-found genes were screened out, which might play a role in the oncogenesis and tumor progression, and be the tumor-related risk factors.

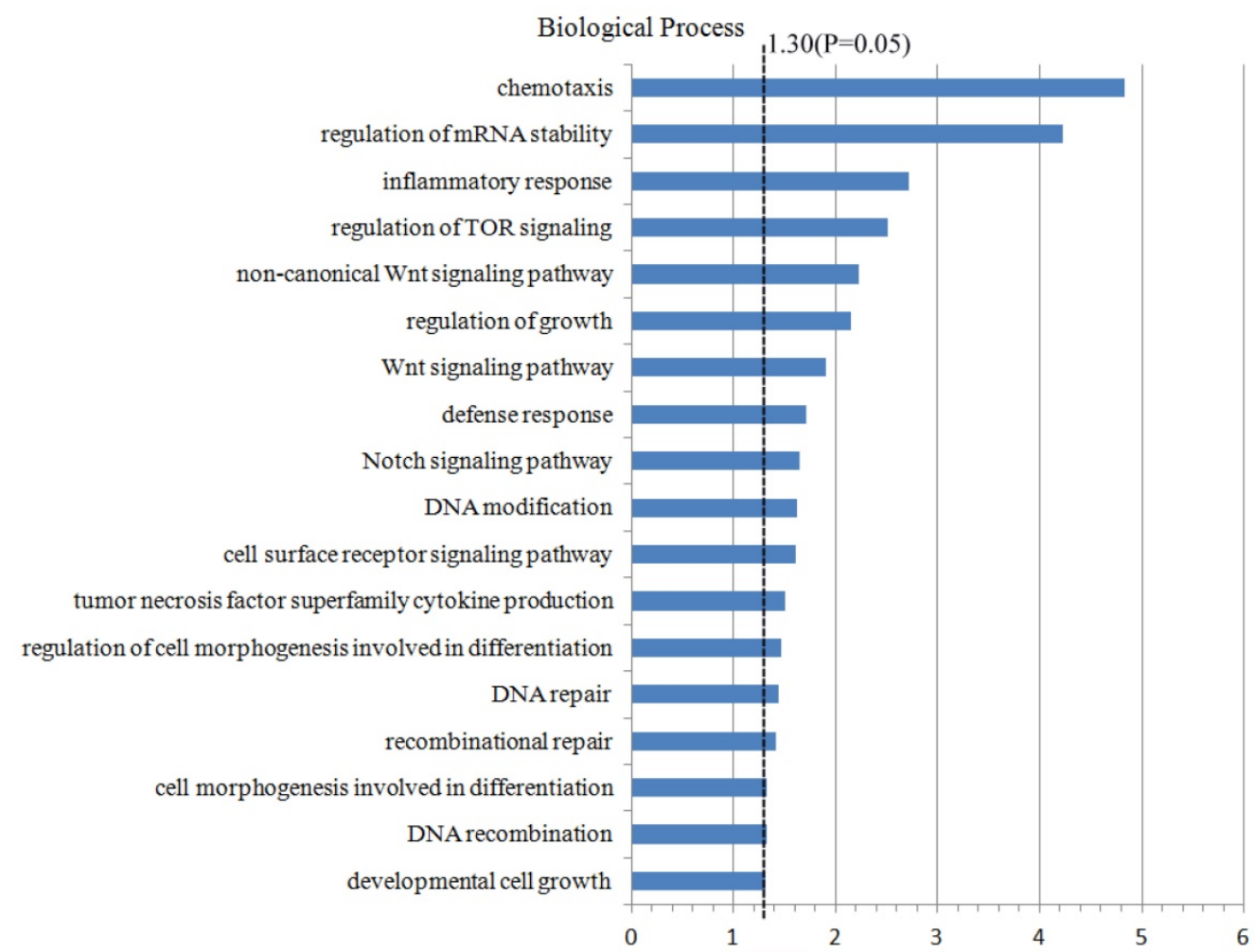

Figure 3. Genes functional annotation and enrichment. 4437 genes which were located in the CNA regions with the frequency $\geq 10 \%$ were included in the Database for Annotation, Visualization and Integrated Discovery to analyze genes annotation and enrichment.

Table 3. $\mathrm{CN}$ status of the genes in different pathways that are significantly enriched

\begin{tabular}{|c|c|c|c|c|c|}
\hline \multirow[t]{2}{*}{ Pathways } & \multirow{2}{*}{ Related genes } & \multicolumn{2}{|c|}{ Chinese } & \multicolumn{2}{|l|}{ TCGA } \\
\hline & & Event & Frequency & Event & Frequency \\
\hline \multirow[t]{6}{*}{ Regulation of mRNA stability } & EXOSC7 & Del & $71.43 \%$ & Del & $10 \%$ \\
\hline & PRKCD & Del & $71.43 \%$ & Del & $9 \%$ \\
\hline & TRIM71 & Del & $68.57 \%$ & Del & $10 \%$ \\
\hline & HNRNPA0 & Amp & $45.71 \%$ & Amp & $13 \%$ \\
\hline & METTL3 & Del & $11.42 \%$ & - & - \\
\hline & PSME1 & Del & $11.42 \%$ & - & - \\
\hline \multirow[t]{12}{*}{ DNA repair } & DDB2 & Amp & $31.43 \%$ & - & - \\
\hline & PARP2 & Del & $11.42 \%$ & - & - \\
\hline & TTC5 & Del & $11.42 \%$ & - & - \\
\hline & APEX1 & Del & $11.42 \%$ & - & - \\
\hline & SUPT16H & Del & $11.42 \%$ & - & - \\
\hline & REC8 & Del & $11.42 \%$ & - & - \\
\hline & FAN1 & Amp & $31.43 \%$ & - & - \\
\hline & EGFR & Amp & $37.14 \%$ & Del & $0.20 \%$ \\
\hline & KIF22 & $\mathrm{LOH}$ & $34.29 \%$ & Amp & $0.20 \%$ \\
\hline & PPP4C & $\mathrm{LOH}$ & $48.57 \%$ & Amp & $0.20 \%$ \\
\hline & INO80E & $\mathrm{LOH}$ & $42.86 \%$ & Amp & $0.20 \%$ \\
\hline & PAGR1 & $\mathrm{LOH}$ & $34.29 \%$ & Amp & $0.20 \%$ \\
\hline \multirow[t]{5}{*}{ DNA modification } & DNMT3A & Amp & $11.42 \%$ & - & - \\
\hline & EHMT2 & Amp & $11.42 \%$ & - & - \\
\hline & ASCC1 & Del & $11.42 \%$ & - & - \\
\hline & CCNB1IP1 & Del & $11.42 \%$ & - & - \\
\hline & CTCF & Del & $11.42 \%$ & - & - \\
\hline \multirow[t]{2}{*}{ Notch signaling pathway } & MDK & Amp & $31.43 \%$ & - & - \\
\hline & MMP14 & Del & $11.42 \%$ & - & - \\
\hline
\end{tabular}




\begin{tabular}{|c|c|c|c|c|c|}
\hline \multirow[t]{2}{*}{ Pathways } & \multirow[t]{2}{*}{ Related genes } & \multicolumn{2}{|c|}{ Chinese } & \multicolumn{2}{|l|}{ TCGA } \\
\hline & & Event & Frequency & Event & Frequency \\
\hline \multirow{4}{*}{ TOR signaling pathway } & FOXA1 & Del & $11.42 \%$ & - & - \\
\hline & TSC2 & Del & $14.29 \%$ & Amp & $0.20 \%$ \\
\hline & TELO2 & $\mathrm{LOH}$ & $11.43 \%$ & Amp & $0.20 \%$ \\
\hline & WDR24 & $\mathrm{LOH}$ & $11.43 \%$ & Amp & $0.20 \%$ \\
\hline \multirow{4}{*}{ Wnt signaling pathway } & MLST8 & Del & $14.29 \%$ & Amp & $0.20 \%$ \\
\hline & PSMB5 & Del & $11.42 \%$ & - & - \\
\hline & PSMB11 & Del & $11.42 \%$ & - & - \\
\hline & PSME2 & Del & $11.42 \%$ & - & - \\
\hline \multirow[t]{5}{*}{ Tumor necrosis factor superfamily } & PYCARD & $\mathrm{LOH}$ & $65.71 \%$ & Amp & $0.20 \%$ \\
\hline & GHRL & Del & $68.57 \%$ & Del & $11 \%$ \\
\hline & CCR2 & Del & $74.29 \%$ & Del & $10 \%$ \\
\hline & MYD88 & Del & $71.43 \%$ & Del & $10 \%$ \\
\hline & LTF & Del & $74.29 \%$ & Del & $11 \%$ \\
\hline \multirow[t]{9}{*}{ Inflammatory response } & TUSC2 & Del & $68.57 \%$ & Del & $10 \%$ \\
\hline & PPARG & Del & $68.57 \%$ & Del & $11 \%$ \\
\hline & IL17RC & Del & $71.43 \%$ & Del & $11 \%$ \\
\hline & CCRL2 & Del & $74.29 \%$ & Del & $11 \%$ \\
\hline & HIF1A & Del & $14.29 \%$ & Del & $0.60 \%$ \\
\hline & LTB4R & Del & $11.42 \%$ & - & - \\
\hline & IL25 & Del & $11.42 \%$ & - & - \\
\hline & LTB4R2 & Del & $11.42 \%$ & - & - \\
\hline & NFATC4 & Del & $11.42 \%$ & - & - \\
\hline
\end{tabular}

CN, copy number; TCGA, the Cancer Genome Atlas; Del, delete; LOH, loss of heterozygosity; Amp, amplification.

\section{Oncogenes, Tumor suppressor genes and Fusion genes}

Through much deeper analyzing 4437 genes which were located in the CNA regions (the frequency $\geq 10 \%$ ), we explored and selected out 23 tumor suppressor genes located in $\mathrm{CN}$ loss regions (TADA3, VHL, PLCD1, CSRNP1, LZTFL1, LRRC2, NAT6, TUSC2, RASSF1, PCBP4, BAP1, ERC2-IT1, PTPRG, FOXP1, etc.), and 10 oncogenes (ABL2, RAB10, ADGRA3, BRAF, MET, LYN, MOS, RAB2A, RNF139, HUWE1) which were located in $\mathrm{CN}$ gain regions (Table 4). There were still no studies on these functional genes associated with ccRCC, except for three genes (VHL, BAP1, MET). Besides, among these functional genes, the CNA of tumor suppressor gene (ERC2-IT1) and oncogenes (RAB10, ADGRA3) were not reported either in TCGA database. Interestingly, except for some higher-frequency genes and no-reporter genes, the opposite results of some CNA genes between Oncoscan assay and TCGA were also noticed, such as TSC2, RNF40 and HUWE1.

To explore the fusion genes, we chose a few high-frequency $\mathrm{CN}$ loss regions for further analyzing, such as 3p, 3q25.33-q26.1 and 22q11.23. Although we did not find the phenomenon of gene fusion in $3 p$ and 3q25.33-q26.1, we observed the relatively highfrequency gene fusion in 22q11.23 (Fig. 4). When the $\mathrm{CN}$ loss of certain fragment in 22q11.23 occurred, two genes fusion (GSTTP1 and GSTTP2) which were located at two sides of $\mathrm{CN}$ loss fragment happened. And in 35 samples, gene fusion occurred in 16 samples $(46 \%)$.
Table 4. Tumor suppressors and Oncogenes associated with cCRCC in this study

\begin{tabular}{|c|c|c|c|c|c|c|}
\hline \multirow[t]{2}{*}{ Function } & \multirow[t]{2}{*}{ Genes } & \multirow[t]{2}{*}{ Region } & \multicolumn{2}{|c|}{ Chinese } & \multicolumn{2}{|l|}{ TCGA } \\
\hline & & & Event & Frequency & Event & Frequency \\
\hline \multirow{23}{*}{$\begin{array}{l}\text { Tumor } \\
\text { suppressors(23) }\end{array}$} & TADA3 & $3 p 25.3$ & Del & $71.43 \%$ & Del & $11.00 \%$ \\
\hline & VHL* & $3 p 25.3$ & Del & $71.43 \%$ & Del & $11.00 \%$ \\
\hline & PLCD1 & $3 p 22.2$ & Del & $71.43 \%$ & Del & $11.00 \%$ \\
\hline & CSRNP1 & $3 p 22.2$ & Del & $71.43 \%$ & Del & $11.00 \%$ \\
\hline & LZTFL1 & 3p21.31 & Del & $74.29 \%$ & Del & $11.00 \%$ \\
\hline & LRRC2 & $3 p 21.31$ & Del & $74.29 \%$ & Del & $11.00 \%$ \\
\hline & SEMA3B & 3 p21.31 & Del & $66.57 \%$ & Del & $11.00 \%$ \\
\hline & NAT6 & $3 p 21.31$ & Del & $68.57 \%$ & Del & $11.00 \%$ \\
\hline & TUSC2 & 3p21.31 & Del & $68.57 \%$ & Del & $11.00 \%$ \\
\hline & RASSF1 & $3 p 21.31$ & Del & $68.57 \%$ & Del & $11.00 \%$ \\
\hline & PCBP4 & $3 p 21.2$ & Del & $71.43 \%$ & Del & $11.00 \%$ \\
\hline & BAP1* & $3 p 21.1$ & Del & $71.43 \%$ & Del & $10.00 \%$ \\
\hline & ERC2-IT1 & $3 \mathrm{p} 14.3$ & Del & $71.43 \%$ & - & - \\
\hline & PTPRG & 3p14.2 & Del & $57.14 \%$ & Del & $5.00 \%$ \\
\hline & FOXP1 & 3p13 & Del & $42.86 \%$ & Del & $2.70 \%$ \\
\hline & RBM5 & $3 p 21.31$ & Del & $68.57 \%$ & Del & $10 \%$ \\
\hline & LATS2 & $13 q 12.11$ & Del & $17.14 \%$ & Del & $0.20 \%$ \\
\hline & RNF6 & $13 q 12.13$ & Del & $17.14 \%$ & Del & $0.20 \%$ \\
\hline & DLK1 & $14 q 32.2$ & Del & $11.43 \%$ & Del & $0.40 \%$ \\
\hline & MEG3 & $14 \mathrm{q} 32.2$ & Del & $11.43 \%$ & Del & $0.40 \%$ \\
\hline & TSC2 & $16 \mathrm{p} 13.3$ & Del & $14.29 \%$ & Amp & $0.20 \%$ \\
\hline & RNF40 & $16 \mathrm{p} 11.2$ & $\mathrm{LOH}$ & $57.14 \%$ & Amp & $0.20 \%$ \\
\hline & STK11 & $19 \mathrm{p} 13.3$ & Del & $20.00 \%$ & Del & $0.20 \%$ \\
\hline \multirow[t]{10}{*}{ Oncogenes (10) } & ABL2 & $1 \mathrm{q} 25.2$ & Amp & $14.29 \%$ & Amp & $0.80 \%$ \\
\hline & RAB10 & $2 \mathrm{p} 23.3$ & Amp & $11.43 \%$ & - & - \\
\hline & ADGRA3 & $4 p 15.2$ & Amp & $11.43 \%$ & - & - \\
\hline & BRAF & $7 q 34$ & Amp & $17.14 \%$ & Amp & $0.90 \%$ \\
\hline & $\mathrm{MET}^{*}$ & $7 q 31.2$ & Amp & $28.57 \%$ & Amp & $0.90 \%$ \\
\hline & LYN & $8 \mathrm{q} 12.1$ & Amp & $14.29 \%$ & Amp & $0.40 \%$ \\
\hline & MOS & $8 \mathrm{q} 12.1$ & Amp & $14.29 \%$ & Amp & $0.40 \%$ \\
\hline & RAB2A & $8 \mathrm{q} 12.1$ & Amp & $14.29 \%$ & Amp & $0.40 \%$ \\
\hline & RNF139 & $8 \mathrm{q} 24.13$ & Amp & $14.29 \%$ & Amp & $0.80 \%$ \\
\hline & HUWE1 & Xp11.22 & Amp & $11.43 \%$ & Del & $0.60 \%$ \\
\hline
\end{tabular}

*: The genes associated with renal cancer have been reported in previous studies; -: not available; ccRCC, clear cell renal cancer; TCGA, the Cancer Genome Atlas; Del, delete; $\mathrm{LOH}$, loss of heterozygosity; Amp, amplification. 


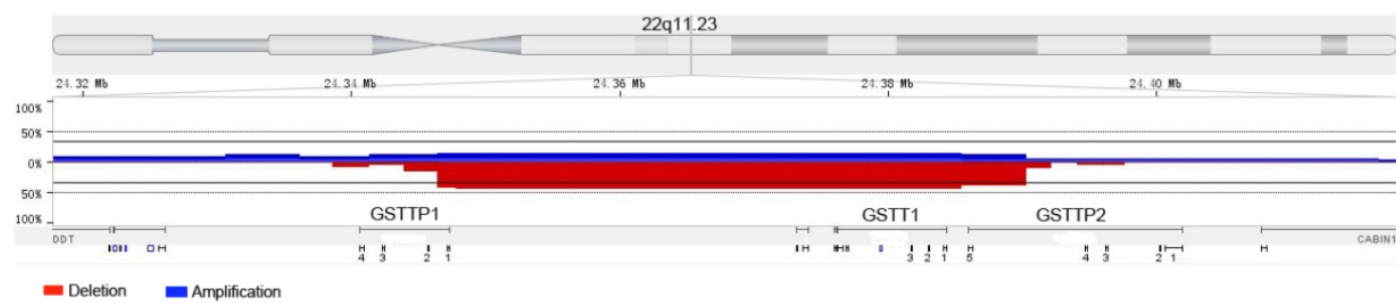

Figure 4. The relatively high-frequency gene fusion in $22 q 11.23$. The red and blue areas respectively represented copy number loss and copy number gain. Two genes fusion (GSTTP1 and GSTTP2) located at two sides of copy number loss fragment were observed.

Table 5. CNA burden in ccRCC patients.

\begin{tabular}{|c|c|c|c|c|}
\hline Event & Average level (\%) & No. of cases & AN (n, \%) & \\
\hline & & $\mathrm{T} 1(\mathrm{n}=28)$ & $\mathrm{T} 2+\mathrm{T} 3(\mathrm{~N}=7)$ & P-value \\
\hline Amplification & $2.91 \%$ & $13(46.43 \%)$ & $4(57.14 \%)$ & 0.69 \\
\hline Deletion & $2.49 \%$ & $13(46.43 \%)$ & $4(57.14 \%)$ & 0.69 \\
\hline Amplification and deletion & $5.40 \%$ & $13(46.43 \%)$ & $7(100 \%)$ & $0.012^{*}$ \\
\hline Loss of heterozygosity & $5.08 \%$ & $13(46.43 \%)$ & $4(57.14 \%)$ & 0.69 \\
\hline
\end{tabular}

CNA, copy number alteration; ccRCC, clear cell renal cancer; *: P-value $<0.05$.

\section{The comparison between different $T$ stages affected by CNA}

In the Table 5, the statistical difference among different $\mathrm{T}$ stages was calculated on some highfrequency CNA regions. Although there were no significance in the group of $\mathrm{CN}$ loss, $\mathrm{CN}$ gain, $\mathrm{LOH}$ (loss of heterozygosity), the significant difference of total CNA between $\mathrm{T} 1$ group and $\mathrm{T} 2+\mathrm{T} 3$ group was noticed $(P$-value $=0.012)$, which mean $\mathrm{T} 2+\mathrm{T} 3$ group carried more high-frequency CNA regions (Supplementary Table 2). In the Supplementary Table 3, we calculated the number of genes affected by CNA in every $\mathrm{T}$ stage patient, namely gene burden, and compared the difference between $\mathrm{T} 1$ and $\mathrm{T} 2+\mathrm{T} 3$ stage. But there were no significance in the group of $\mathrm{CN}$ loss, $\mathrm{CN}$ gain, $\mathrm{LOH}$, and total CNA $(P$-value $>0.05)$. As the Supplementary Table 4 showed, the affected bp per $\mathrm{Mbp}$ regions in the whole genome (about $3000 \mathrm{Mbp}$ ) of every patient were analyzed, and the significance on different $\mathrm{T}$ stages in the group of $\mathrm{CN}$ loss, $\mathrm{CN}$ gain, $\mathrm{LOH}$, and total CNA were not found out ( $P$-value $>$ 0.05).

\section{Discussion}

As far as we know, it is the first time that the whole-genome CNA in ccRCC was reported in the Chinese population, and the landscape of wholegenome CNA in ccRCC was acquired. In this study, we found out a few CNA regions which were not reported in previous studies, and discovered some new functional genes associated with ccRCC which had definite biological meaning. For example, the $\mathrm{CN}$ loss of tumor suppressor genes and DNA repair genes, the $\mathrm{CN}$ gain of oncogenes, fusion genes, etc. Commonly, gene dose effect, loss of gene function, and gene fusion were three likely mechanisms in CNA that would lead to the disease [7]. Therefore, these functional genes had potential to become the biomarkers of ccRCC, and were used for early diagnosis and guidance of follow-up.

In previous studies, researchers acquired the somatic CNAs by detecting 385 ccRCC samples with gene sequencing [8]. They discovered a few highfrequency CNA regions and functional genes, for example, the $\mathrm{CN}$ loss in 3p21.1, 14q24, 9p21.3 (CDKN2A), 6q26 (QKI), 8p11, 10q23 (PTEN), 1p26, 4q35, 13q21 (RB1), 15q21, 2q37, and the $\mathrm{CN}$ gain in 5q35, 8q24 (MYC), 3q26 (MECOM), 1q32 (MDM4). It was supposed that $\mathrm{CN}$ loss of tumor suppressor genes (CDKN2A, PTEN, RB1) and CN gain of oncogene (MYC) could be closely associated with tumorigenesis $[8,9]$. In another study, it reported some highfrequency CNA regions and functional genes by whole-genome sequencing in 85 ccRCC samples, for example, EPM2AIP1 and SUSD5 at 3p22-p24, APEH, SETD2 and SMARCC at 13p21.31, SLC6A6 and VHL at 3p25, YLPM1 at 14q24.3, C14orf39 at 14q23, PNLIP at $10 \mathrm{q} 25$, DOCK2 at 5q35.1, KAT5 at 11q13.1, CHRNB2 and ECM1 at 1q21.2, AKAP9 and MUC17 at $7 q 11-q 22$ [10]. It was reported that ccRCC was characterized by loss of function of the tumor suppressor VHL and activation of hypoxia-inducible transcription factors (HIFs), and the VHL gene product $(\mathrm{pVHL})$ targeted HIFs transcription factors for proteasomal degradation, and then increased HIF2a promotes pVHL-defective tumorigenesis [11-13]. There was a study that showed $5 \mathrm{q}$ amplification led to overexpression of the SQSTM1 oncogene in ccRCC lines and tumors, and the gene product of which, p62, regulated known renal cancer suppressor genes such as VHL, TSC1and TSC2 
$[14,15]$. Therefore, SQSTM1 was regarded as a pathogenic target of $5 \mathrm{q} \mathrm{CN}$ gains in renal cancer. Besides, there were studies suggested that the $\mathrm{CN}$ loss of tumor suppressor CDKNA2 at $9 p$ and the $\mathrm{CN}$ gain of oncogene MET at 7q31.1 were closely related to RCC, having the potential predictive value to guide targeted therapy $[16,17]$.

In the Table 3, we listed the new-found functional genes that were not reported before in ccRCC, including DNA repair genes, DNA modification genes, Notch signal pathway genes, and Wnt signal pathway genes. The previous studies validated that the alterations in DNA repair genes were closely associated with tumorigenesis and prognosis, including renal cancer [18,19]. In DNA modification genes we found, several studies showed that CN loss of CTCF at 16q22.1 and its low expression occurred in breast cancer and Wilms tumor of the kidney $[20,21]$. A study demonstrated that CNA led to Notch pathway activation in ccRCC, and relevant gene products were also overexpressed and associated with gene amplification in distinct ccRCC samples [22]. The high-frequency $\mathrm{CN}$ gain of MDK at 11 p11.2 in our study might validate these outcomes. Moreover, another study indicated that lower FOXA1 expression might be a marker of aggressive disease in upper tract urothelial carcinoma undergoing radical nephroureterectomy [23]. This outcome might be an evidence to prove that $\mathrm{CN}$ loss of FOXA1 at 14q11.2 was associated with ccRCC in our study. There was a research indicated that the low expression of the Wnt signal pathway gene (Wnt7A) was detected in the majority of ccRCC, and might have tumor suppressor properties [24]. Therefore, this result might be also a proof to verify that $\mathrm{CN}$ loss of PSME2, PSMB11, PSMB5 was associated with ccRCC in our study.

In the Table 4, we made a list of functional genes, including 23 tumor suppressor genes located in $\mathrm{CN}$ loss regions and 10 oncogenes at $\mathrm{CN}$ gain regions. The previous studies had validated that $\mathrm{CN}$ loss of tumor suppressors VHL, BAP1, and CN gain of oncogene MET might play a role in tumorigenesis and progression $[16,25]$. For the rest of genes listed in Table 4, there were still no studies on ccRCC CNA in these functional genes. In short, all they had the potential to become markers of ccRCC. For the fusion genes, although both GSTTP1 and GSTTP2 were pseudogene, GSTTP1 still took part in Glutathione metabolism. As we know, the metabolic change was one of the important characters of tumor cells. Moreover, more and more researches revealed that pseudogene could regulate normal genes or express a part of protein, and the alteration in pseudogene might result in cancer [26]. Actually, we should further design the primers corresponding to GSTTP1 and GSTTP2, to validate the existing of gene fusion by PCR test.

In addition, the CNA frequency of some regions in Chinese was obviously higher when compared with TCGA database. Some genes which were found to be deleted in the TCGA database are amplified in our Ocoscan assay (e.g., EGFR, etc.), while some genes which were found to be amplified in the TCGA are deleted in our study (e.g., TSC2, etc.). However, it is hard to conclude whether the discrepancy is indeed due to race (Chinese population versus European Americans) or merely due to small study size. A future larger cohort should also be conducted to validate these findings.

Several limitations of the current study should be noted. (1) The sample size is relatively small in this study. It is hard to conclude whether the discrepancy is indeed due to race (Chinese population versus European Americans) or merely due to small size. It would be more persuasive to conduct Oncoscan assay on more samples. (2) The differences which had CNA regions or genes between different $\mathrm{T}$ stages were not detected, probably because of the small sample size which limited the statistic significance. (3) For the functional genes we found in this study, the validation by low-throughput method (MLPA, HRM-PCR, etc.) should be done in larger scale of samples. (4) The study on expression of functional genes is lacking, we will conduct the related experiment to validate the function of these genes in the next step.

\section{Conclusion}

In this study, we acquired the whole-genome ccRCC CNA landscape in the Chinese population, discovered a few functional genes and fusion genes related to ccRCC in the CNA regions which maybe play a role in the biological process of tumor tumorigenesis. However, the larger scale of samples still is needed to validate our results.

\section{Abbreviations}

RCC: renal cell cancer; ccRCC: clear cell renal cancer; CNA: copy number alteration; bp: base pair; Mbp: million bp; $\mathrm{CN}$ loss: copy number loss; $\mathrm{CN}$ gain: copy number gain; BP: biological process; GO: gene ontology; BMI: body mass index; TCGA: the Cancer Genome Atlas; DAVID: The Database for Annotation, Visualization and Integrated Discovery; Chr: chromosome; Del: delete; LOH: loss of heterozygosity; Amp: amplification.

\section{Supplementary Material}

Supplementary figures and tables. http://www.jcancer.org/v11p0016s1.pdf 


\section{Acknowledgement}

This work was supported by the National Natural Science Foundation of China (81570607) and the three-year action plan for promoting clinical skills and clinical innovation in municipal hospitals of Shanghai Shenkang (16CR2003A).

\section{Competing Interests}

The authors have declared that no competing interest exists.

\section{References}

1. Ljungberg B, Bensalah K, Canfield S, Dabestani S, Hofmann F, Hora M, et al. EAU guidelines on renal cell carcinoma: 2014 update. European urology. 2015; 67: 913-24.

2. Siegel RL, Miller KD, Jemal A. Cancer Statistics, 2017. CA: a cancer journal for clinicians. 2017; 67: 7-30.

3. Chen $\mathrm{W}$, Zheng R, Baade PD, Zhang S, Zeng H, Bray F, et al. Cancer statistics in China, 2015. CA: a cancer journal for clinicians. 2016; 66: 115-32.

4. Arai E, Kanai Y. Genetic and epigenetic alterations during renal carcinogenesis. International journal of clinical and experimental pathology. 2010; 4: 58-73.

5. Stranger BE, Forrest MS, Dunning M, Ingle CE, Beazley C, Thorne N, et al. Relative impact of nucleotide and copy number variation on gene expression phenotypes. Science. 2007; 315: 848-53.

6. Foster JM, Oumie A, Togneri FS, Vasques FR, Hau D, Taylor M, et al. Cross-laboratory validation of the OncoScan(R) FFPE Assay, a multiplex tool for whole genome tumour profiling. BMC medical genomics. 2015; 8: 5.

7. Kuiper RP, Ligtenberg MJ, Hoogerbrugge N, Geurts van Kessel A. Germline copy number variation and cancer risk. Current opinion in genetics \& development. 2010; 20: 282-9.

8. Network CGAR. Comprehensive molecular characterization of clear cell renal cell carcinoma. Nature. 2013; 499: 43-9.

9. O'Leary NA, Wright MW, Brister JR, Ciufo S, Haddad D, McVeigh R, et al. Reference sequence (RefSeq) database at NCBI: current status, taxonomic expansion, and functional annotation. Nucleic acids research. 2016; 44: D733-45.

10. Scelo G, Riazalhosseini Y, Greger L, Letourneau L, Gonzalez-Porta M, Wozniak MB, et al. Variation in genomic landscape of clear cell renal cell carcinoma across Europe. Nature communications. 2014; 5: 5135

11. Damjanovic SS, Ilic BB, Beleslin Cokic BB, Antic JA, Bankovic JZ, Milicevic IT, et al. Tuberous sclerosis complex protein 1 expression is affected by VHL Gene alterations and HIF-1alpha production in sporadic clear-cell renal cell carcinoma. Experimental and molecular pathology. 2016; 101: 323-31.

12. Grampp S, Platt JL, Lauer V, Salama R, Kranz F, Neumann VK, et al. Genetic variation at the $8 \mathrm{q} 24.21$ renal cancer susceptibility locus affects HIF binding to a MYC enhancer. Nature communications. 2016; 7: 13183.

13. Shen C, Kaelin WG, Jr. The VHL/HIF axis in clear cell renal carcinoma. Seminars in cancer biology. 2013; 23: 18-25.

14. Li L, Shen C, Nakamura E, Ando K, Signoretti S, Beroukhim R, et al. SQSTM1 is a pathogenic target of $5 \mathrm{q}$ copy number gains in kidney cancer. Cancer cell. 2013; 24: 738-50.

15. Kozlowski P, Roberts P, Dabora S, Franz D, Bissler J, Northrup H, et al. Identification of 54 large deletions/duplications in TSC1 and TSC2 using MLPA, and genotype-phenotype correlations. Human genetics. 2007; 121: 389-400.

16. Macher-Goeppinger S, Keith M, Endris V, Penzel R, Tagscherer KE, Pahernik $S$, et al. MET expression and copy number status in clear-cell renal cell carcinoma: prognostic value and potential predictive marker. Oncotarget. 2017; 8: 1046-57.

17. El-Mokadem I, Kidd T, Pratt N, Fleming S, Nabi G. Tumour suppressor gene (CDKNA2) status on chromosome $9 p$ in resected renal tissue improves prognosis of localised kidney cancer. Oncotarget. 2016; 7: 73045-54.

18. Margulis V, Lin J, Yang H, Wang W, Wood CG, Wu X. Genetic susceptibility to renal cell carcinoma: the role of DNA double-strand break repair pathway. Cancer epidemiology, biomarkers \& prevention. 2008; 17: 2366-73.

19. Akhmadishina LZ, Giliazova IR, Kutlyeva LR, Korytina GF, Kochetova OV, Urmantsev MF, et al. [DNA repair XRCC1, XPD genes polymorphism as associated with the development of bladder cancer and renal cell carcinoma]. Genetika. 2014; 50: 481-90.

20. Mummert SK, Lobanenkov VA, Feinberg AP. Association of chromosome arm $16 \mathrm{q}$ loss with loss of imprinting of insulin-like growth factor-II in Wilms tumor. Genes, chromosomes \& cancer. 2005; 43: 155-61.

21. Green AR, Krivinskas S, Young P, Rakha EA, Paish EC, Powe DG, et al. Loss of expression of chromosome 16q genes DPEP1 and CTCF in lobular carcinoma in situ of the breast. Breast cancer research and treatment. 2009; 113: 59-66.
22. Bhagat TD, Zou Y, Huang S, Park J, Palmer MB, Hu C, et al. Notch Pathway Is Activated via Genetic and Epigenetic Alterations and Is a Therapeutic Target in Clear Cell Renal Cancer. The Journal of biological chemistry. 2017; 292: 837-46.

23. Raman JD, Warrick JI, Caruso C, Yang Z, Shuman L, Bruggeman RD, et al. Altered Expression of the Transcription Factor Forkhead Box A1 (FOXA1) Is Associated With Poor Prognosis in Urothelial Carcinoma of the Upper Urinary Tract. Urology. 2016; 94: 314 e1-7.

24. Kondratov AG, Kvasha SM, Stoliar LA, Romanenko AM, Zgonnyk YM, Gordiyuk VV, et al. Alterations of the WNT7A gene in clear cell renal cell carcinomas. PloS one. 2012; 7: e47012.

25. Gao W, Li W, Xiao T, Liu XS, Kaelin WG, Jr. Inactivation of the PBRM1 tumor suppressor gene amplifies the HIF-response in VHL-/- clear cell renal carcinoma. Proceedings of the National Academy of Sciences of the United States of America. 2017; 114: 1027-32.

26. Hirotsune S, Yoshida N, Chen A, Garrett L, Sugiyama F, Takahashi S, et al. An expressed pseudogene regulates the messenger-RNA stability of its homologous coding gene. Nature. 2003; 423: 91-6. 